\title{
The Focused Beam Forming with a Monolithic 2D Phased Array
}

\author{
B. LILA* AND J. KAPELEWSKI \\ Electronics Department, Military University of Technology \\ S. Kaliskiego 2, 00-908 Warsaw, Poland
}

\begin{abstract}
A new type of the monolithic acoustic scanner based on a near-surface quasicomposite two-dimensional array, implemented in the solid elastic substrate was proposed. It provides a simple means of focusing and scanning a beam of acoustic waves, radiated into the depth of the elastic solid substrate. A chirped surface acoustic wave is converted into a focused bulk wave by scattering from an array composed of a $2 \mathrm{D}$ periodic system of regions with varying material parameters, located at the surface of a surface acoustic waves substrate. In the paper, a simple numerical simulation model is presented to analyse the influences of the dynamical and geometrical parameters of the surface structure, as well as the influences of incident surface acoustic waves signal profile on the focal distribution.
\end{abstract}

PACS numbers: 46.40.-f, 43.58.+z

\section{Introduction}

Acoustic imaging systems operating at high frequencies have been important for the nondestructive testing (NDT) of structural materials, biomedical imaging and microelectronics [1-3]. Most of the present imaging devices use the $1 \mathrm{D}$ or 2D phased array technique, where the phased array is a multi-element piezoelectric device, the elements of which are individually excited by electric pulses at programmed delay times.

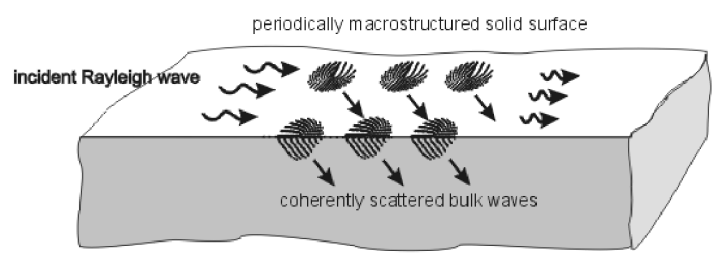

Fig. 1. An illustration of the scattering process at the $2 \mathrm{D}$ periodic surface array.

*corresponding author; e-mail: blila@wat.edu.pl 
These solutions have many technological problems in fabrication, associated mainly with the number of microelements required. Apart from this, the upper range of working frequencies is strongly limited by piezoelectric lead zirconate titanate (PZT)-type materials which have been used in mentioned "classic" composite arrays. The possibility of the grating acoustic scanner (GAS) using as an electronically focused and scanned transmitter and receiver for acoustic imaging have been previously shown in $[4,5]$. Such grating scanner relied on mode conversion of surface acoustic waves (SAW) to bulk acoustic waves (BAW) for the generating of directed acoustic energy beam.

In Refs. [6, 7] we have proposed an alternative method of mode conversion, employing array composed of a two-dimensional (2D) periodic system of regions with varying material parameters or, alternatively, altered phase inclusions, located at the surface of a SAW substrate (Fig. 1). The time delay required for steering and focusing the excited beam is then provided by the incident surface wave propagating through the array along chosen directions. One way to produce such an array, being in fact a kind of composite-like surface structure, is by using the ion implantation or the metal impurity selective diffusion technique, with making use of a suitable doping window [6, 7]. An important advantage of such a technique for surface modification is a possibility to adjust both the length of the implanted acoustic path, and the basic implantation parameters. It is of importance for performing the optimised trade-off of the change in characteristics of the SAW into the BAW conversion process. For periodic structures, an energy focusing may be effectively realised by using "chirp" SAW pulse signal as incident wave [7] (Fig. 2).

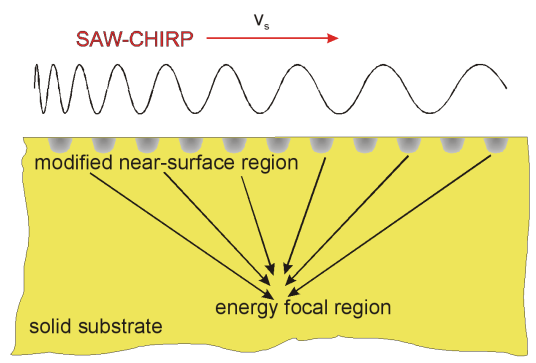

Fig. 2. Bulk-wave focusing by scattering chirped-SAW from a periodic surface array.

\section{Basic equations of the focusing mechanism}

For the case in consideration, with a one incident surface acoustic wave of the Rayleigh type propagating along the one of the 2D surface structure symmetry axis, we can describe the relations between the chirp SAW parameters, geometry of the structure and substrate material parameters, by using similar equations, to these presented in [3] for the grating scanner. In this way, the focal length and 
the focused beam width are given by

$$
h=\frac{v_{R}}{v_{l}} \frac{f_{0}}{B} W, \quad s=\frac{W}{\Delta T B}=\lambda_{l} \frac{h}{W},
$$

where $h$ - focal length, $s-4 \mathrm{~dB}$ beam width, $\Delta T$ - chirp length, $B$ - chirp band width, $W=v_{R} \Delta T$ - chirp spatial width, $v_{l}$ - longitudinal bulk wave velocity inside the solid substrate, $v_{R}-\mathrm{SAW}$ velocity on the surface periodic structure, $\lambda_{l}$ — wavelength of the longitudinal bulk wave, and $f_{0}$ - centre frequency of chirp.

\section{Numerical simulation}

The model treatment employed here is simulated on the basis of the Huyghens superposition principle, combined with the variational-perturbative treatment $[8,9]$. It describes the dynamic stress distribution on the $2 \mathrm{D}$ periodically modified surface as a set of planar equivalent radiating sources. The scattered waves radiating from equivalent surface point sources into the depth of the solid substrate, are taken to be linear frequency modulated signals with the Hanning window. Therefore, the ultrasonic wave generated from an each equivalent surface point propagates into a solid substrate in a shape of a half-circle, the centre of which is located just in this point. In this study, the focusing process simulation was performed for two variants. In the first, the solid substrate was treated as an isotropic one, with SAW velocity taken as for a real lithium niobiate, but with the bulk longitudinal wave taken as averaged constant value in all propagation directions. In the second, the model was constructed for real anisotropic $Y$-cut $Z$ propagating $\mathrm{LiNbO}_{3}$ with taking bulk acoustic wave velocities for various orientation into account (Fig. 3). The simulated acoustic scanner based on the monolithic quasicomposite phased array is characterised by data presented in Table. The direction of propagation is specified by the polar angle $\theta$ between $Z$-axis and the propagation direction.

TABLE

Data for simulated acoustic scanner.

\begin{tabular}{l|c}
\hline \multicolumn{1}{c|}{ Crystal } & $\mathrm{LiNbO}_{3}$ \\
\hline clusters structure periodicity & $34.8 \mu \mathrm{m}$ \\
synchronous frequency for the clusters structure & $100 \mathrm{MHz}$ \\
fractional band width & from $75 \mathrm{MHz}$ to $125 \mathrm{MHz}$ \\
approximate resolution & $69.6 \mu \mathrm{m}$ \\
number of clusters in the one of 2D structure axes & 130 \\
chirp length & $1.3 \mu \mathrm{s}$ \\
approximate focus value & $4.313 \mathrm{~mm}$ \\
a volume of the 2D mapping mesh of the simulated & $520 \times 1040 \mathrm{nodes}$
\end{tabular}




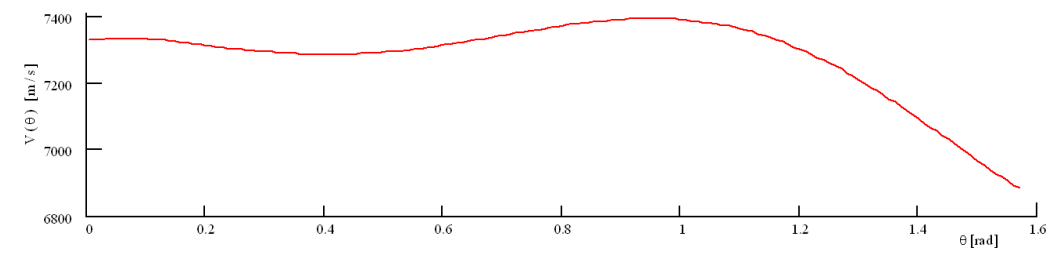

Fig. 3. A velocity of a bulk quasilongitudinal acoustic wave propagating in the $Y Z$ -plane of an $\mathrm{LiNbO}_{3}$ crystal, depending on direction of propagation (an approximation based on a data presented in [9]).

\section{Results and discussion}

In the model treated, the ultrasonic field of SAW-BAW scanner can be evaluated for any position and time. The acoustic field can be visualised by mapping the obtained amplitude into a colour-scale image. Figure 4 shows snapshots of propagating longitudinal bulk wave focused, for parameters assumed from Table presented above. As it can be clearly observed, an anisotropy influence brings a

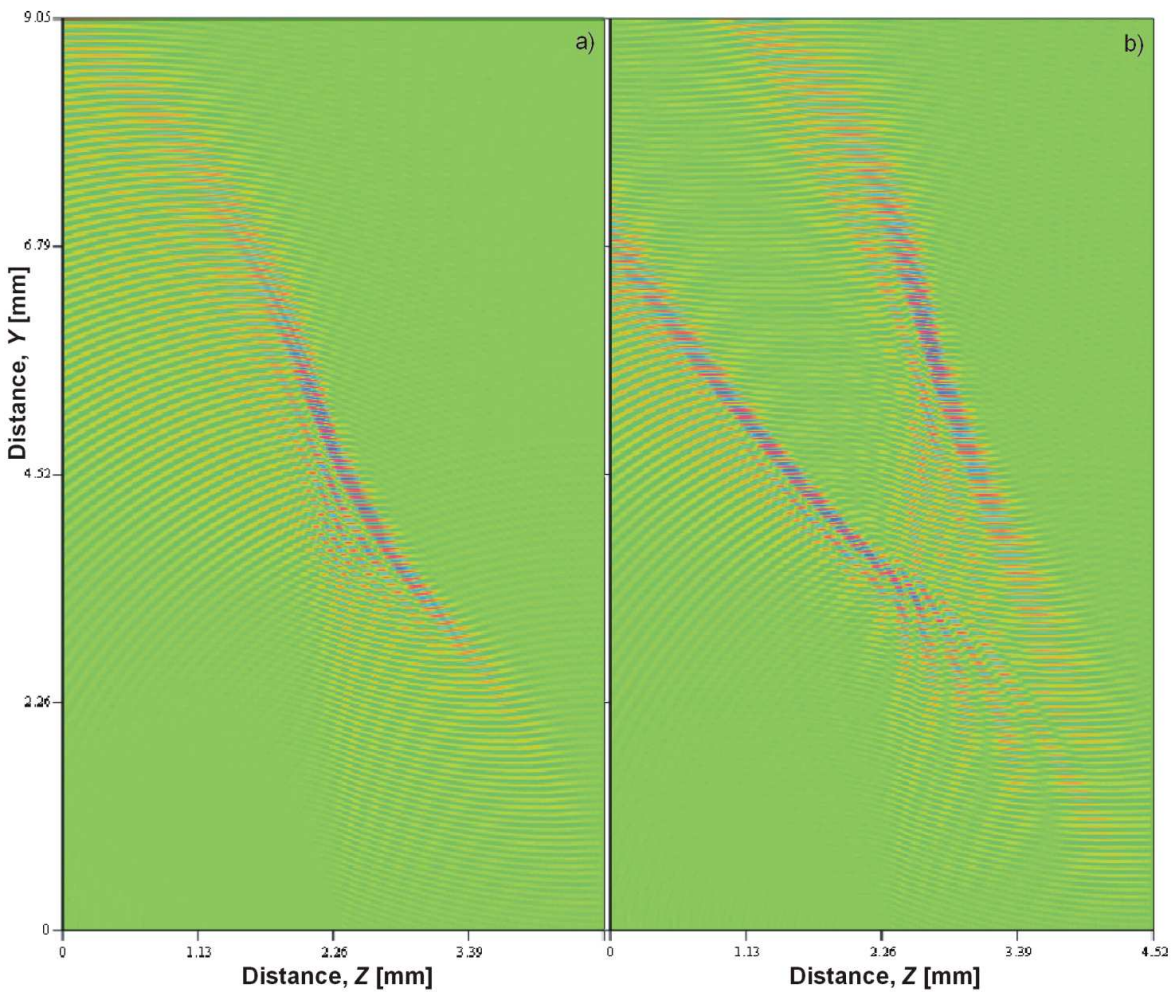

Fig. 4. A snapshot of propagating longitudinal bulk wave focused, for parameters presented in Table; (a) an isotropic approximation, (b) an acoustic field computed with using a relation represented by diagram in Fig. 3 . 
focused acoustic energy splitting effect. These results show that the linear chirp modulation of the SAW signal is only moderately effective for scanners based on anisotropic crystals. For optimal energy focusing in devices of this type, authors of $[7,10,11]$ recommend non-linear chirped SAW.

The both simulations were computed for $t=t_{\mathrm{opt}}$, where $t_{\mathrm{opt}}$ represents the time value, for wich the focusing effect achieves a maximum of an acoustic energy local concentration in a material substrate.

\section{References}

[1] B. Nongaillard, J.M. Rouvaen, E. Bridoux, R. Torguet, C. Bruneel, J. Appl. Phys. 1245-1249, 50 (1979).

[2] H. Takeuchi, C. Nakaya, K. Katakura, in: IEEE Ultrason. Symp., Ed. B.R. McAvoy, Institute of Electrical and Electronic Engineers, New York 1984, p. 507.

[3] W.H. Chen, A. Ronnekleiv, H.J. Shaw, J. Souquet, L.T. Zitelli, in: IEEE Ultrason. Symp., Eds. J. deKlerk, B.R. McAvoy, Institute of Electrical and Electronic Engineers, New York 1977, p. 278.

[4] W.H. Chen, H.J. Shaw, L.T. Zitelli, in: IEEE Ultrason. Symp., 1978, p. 775.

[5] J. Kapelewski, M. Pasternak, Composite Interfaces 3, 5/6 (1996).

[6] J. Kapelewski, B. Lila, in: ICCE/10 Proc., New Orleans 2003, Ed. D. Hui, International Community for Composites Engineering, New Orleans 2003, p. 305.

[7] W.H. Chen, F.C. Fu, W.L. Lu, IEEE Trans. Sonics Ultrason. SU-32, 2 (1985).

[8] P. Kaniewski, Mol. Quant. Acoust. 28, 151 (2007).

[9] P. Kaniewski, Mol. Quant. Acoust. 28, 165 (2007).

[10] J. Kapelewski, B. Lila, A. Dukata, Bull. MUT LV, 53 (2000) (in Polish).

[11] D. Craig, Acoustic Wave Propagation and Properties in Properties of Lithium Niobiate Proc., Ed. K.K. Wong, INSPEC, London 2002, Ch. 10. 